\title{
Homology-directed gene-editing approaches for hematopoietic stem and progenitor cell gene therapy
}

\author{
Manoj Kumar K. Azhagiri 1,2, Prathibha Babu ${ }^{1,2}$, Vigneshwaran Venkatesan ${ }^{1,2}$ and Saravanabhavan Thangavel ${ }^{1 *}$ (D)
}

\begin{abstract}
The advent of next-generation genome engineering tools like CRISPR-Cas9 has transformed the field of gene therapy, rendering targeted treatment for several incurable diseases. Hematopoietic stem and progenitor cells (HSPCs) continue to be the ideal target cells for gene manipulation due to their long-term repopulation potential. Among the gene manipulation strategies such as lentiviral gene augmentation, non-homologous end joining (NHEJ)-mediated gene editing, base editing and prime editing, only the homology-directed repair (HDR)-mediated gene editing provides the option of inserting a large transgene under its endogenous promoter or any desired locus. In addition, HDR-mediated gene editing can be applied for the gene knock-out, correction of point mutations and introduction of beneficial mutations. HSPC gene therapy studies involving lentiviral vectors and NHEJ-based gene-editing studies have exhibited substantial clinical progress. However, studies involving HDR-mediated HSPC gene editing have not yet progressed to the clinical testing. This suggests the existence of unique challenges in exploiting HDR pathway for HSPC gene therapy. Our review summarizes the mechanism, recent progresses, challenges, and the scope of HDRbased gene editing for the HSPC gene therapy.
\end{abstract}

Keywords: Hematopoietic stem and progenitor cells, Gene-editing and homology-directed repair

\section{Introduction}

Recent estimates suggest that over 8000 diseases are of monogenic in origin, often manifesting during childhood and causing premature deaths in severe cases. The burden of genetic disorders remains alarmingly high as the clinical management of many such diseases is largely inefficient. For monogenic disorders, such as $\beta$-hemoglobinopathies ( $\beta$-thalassemia and sickle cell disease (SCD)), cystic fibrosis, hemophilia, Huntington's disease and Duchenne muscular dystrophy, targeted therapeutic strategies are in high demand.

\footnotetext{
*Correspondence: sthangavel@cmcvellore.ac.in

${ }^{1}$ Centre for Stem Cell Research (CSCR), a Unit of InStem Bengaluru, Christian Medical College Campus, Vellore, Tamil Nadu, India

Full list of author information is available at the end of the article
}

Gene therapy aims to correct the root cause of monogeneic disorders by directly acting at DNA level and by employing a wide array of viral or nuclease-based strategies such as gene supplementation, silencing, correction or disruption. Gene augmentation using viral vectors is the most clinically advanced strategy; however, this has lately been known to posit risks such as random integration, insertional mutagenesis and immunogenicity [1]. The development of customizable DNA cleaving endonucleases such as meganucleases, zinc finger nucleases (ZFNs), transcription activator-like effector nucleases (TALENs) and clustered regularly interspaced short palindromic repeats-associated RNA-guided Cas9 (CRISPRCas9) revolutionized the field of gene editing and allowed facile gene manipulation.

Nuclease-mediated gene editing is achieved by exploiting the intrinsic DNA repair pathways such as

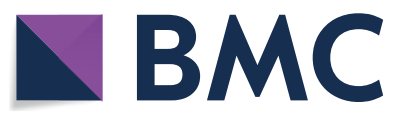

(c) The Author(s) 2021. Open Access This article is licensed under a Creative Commons Attribution 4.0 International License, which permits use, sharing, adaptation, distribution and reproduction in any medium or format, as long as you give appropriate credit to the original author(s) and the source, provide a link to the Creative Commons licence, and indicate if changes were made. The images or other third party material in this article are included in the article's Creative Commons licence, unless indicated otherwise in a credit line to the material. If material is not included in the article's Creative Commons licence and your intended use is not permitted by statutory regulation or exceeds the permitted use, you will need to obtain permission directly from the copyright holder. To view a copy of this licence, visit http://creativecommons.org/licenses/by/4.0/. The Creative Commons Public Domain Dedication waiver (http://creativeco mmons.org/publicdomain/zero/1.0/) applies to the data made available in this article, unless otherwise stated in a credit line to the data. 
non-homologous end-joining (NHEJ) and homologydirected repair (HDR) which are activated following the generation of double-stranded breaks (DSB). The NHEJ repair results in the direct ligation of the cleaved strands producing InDels (insertions-deletions) and is predominantly used for gene disruption, whereas HDR follows a directed correction strategy where an exogenous repair template with the desired nucleotide sequence mediates the process. Thus, the targeted genome-editing strategies hold a great promise for establishing precision edits and can overcome the risks associated with the viral-based strategies [1, 2]. A comparison between gene addition and genome-editing strategies is listed in Table 1.

HSPCs are the ideal targets for the gene therapy of many hematological and immunological disorders. Using HSPCs for gene therapy provides potential long-term benefits as they replenish the patient's hematopoietic system with gene-modified stem cells. Establishing efficient HDR editing in HSPCs is crucial for attaining favorable therapeutic outcomes [3]. This review discusses the applications of HDR in HSPC gene therapy, challenges and the possible solutions.

\section{Homology-directed repair of DNA breaks}

The cell cycle checkpoints and intrinsic DNA repair pathways help to curb the detrimental genomic damages exerted by various genotoxic agents, exogenous nucleases and replication stress. Most genomic lesions are corrected either by NHEJ or HDR pathways, the choice of which is dependent on the DNA resection at DSB site as well as recruitment of repair proteins. The HDR pathway is illustrated in Fig. 1.

53BP1 and BRCA1 are the two key factors that regulates DNA resection. 53BP1 antagonizes the DNA end resection by forming a complex with RIF1 which blocks the BRCA1 recruitment to the DNA DSBs. Thus, the unresected DSBs undergo NHEJ repair pathway which is active throughout interphase. The unresected DSBs also activate ATM checkpoint, coordinating DNA repair and cell cycle. When cells enter S-phase of the cell cycle, CDK-mediated S372 phosphorylation of CtIP activates CtIP and mediates the interaction with BRCA1. BRCA1CtIP complex promotes dephosphorylation and repositioning of 53BP1 creating a chromatin environment for DNA end resection machinery. The BRCA1-CtIP forms a major complex with MRE11-RAD50-NBS1 (MRN) proteins, and this complex generates a short 3'overhanging single-stranded DNA at the DSB ends through the nuclease activity of MRE11. The generated ssDNA is immediately stabilized by replication protein A (RPA), and the short ssDNA is further extended by a helicases and nucleases complex which involves Bloom syndrome protein (BLM) and exonuclease 1 (EXO1) or WRN and DNA replication helicase/nuclease 2 (DNA2). The ssDNA also activates ATR cell cycle checkpoint. The end resection is terminated by BRCA2 mediated RAD51 loading which evicts RPA and forms nucleoprotein filaments with ssDNA. RAD51 nucleoprotein filaments promote homologous search and invasion of the repair template for the start of the repair process [4-6]

The complex events after the DSB vary with the type of HDR-canonical HDR, synthesis-dependent stranded annealing (SDSA), break-induced replication (BIR), single-stranded annealing (SSA) and singlestranded templated repair (SSTR). Although the major steps including end resection, homologous template search, synthesis of new strand from the template, ligation of the DNA ends run similar across all types of HDR, the subsequent steps vary with the type of HDR intended. In canonical HDR, 3' overhang of the ssDNA invades the similar DNA duplex by strand invasion and D loop is formed between the 3' overhang strand and the homologous DNA template. DNA polymerase extends the 3' invading strand, and this changes

Table 1 Comparison of gene addition and genome-editing strategies

\begin{tabular}{|c|c|c|c|}
\hline & $\begin{array}{l}\text { viral-mediated gene addition } \\
\text { strategies }\end{array}$ & $\begin{array}{l}\text { HDR-based genome-editing } \\
\text { strategies }\end{array}$ & $\begin{array}{l}\text { NHEJ-based genome-editing } \\
\text { strategies }\end{array}$ \\
\hline Therapeutic gene expression & $\begin{array}{l}\text { Depending on the vector copy num- } \\
\text { ber and the integration sites }\end{array}$ & $\begin{array}{l}\text { Physiological level expression from } \\
\text { endogenous locus } \\
\text { Supraphysiological levels from safe } \\
\text { harbor, alpha globin locus }\end{array}$ & - \\
\hline Efficiency in human HSPCs & $\begin{array}{l}\text { Depends on transduction efficiency } \\
\text { Transgene, promoter and other regula- } \\
\text { tory sequences in the vector }\end{array}$ & $\begin{array}{l}\text { Depends on frequency of HDR events, } \\
\text { donor types, donor sequences \& avail- } \\
\text { ability at cut site }\end{array}$ & Frequency of productive indels \\
\hline Genotoxic risks & $\begin{array}{l}\text { Oncogene transactivation, generation } \\
\text { of aberrant transcripts, gene inactiva- } \\
\text { tion }\end{array}$ & $\begin{array}{l}\text { Indels at HDR site } \\
\text { Off-targets, large genomic rearrange- } \\
\text { ments }\end{array}$ & $\begin{array}{l}\text { Off-targets, large genomic rear- } \\
\text { rangements }\end{array}$ \\
\hline Costs & High (viral delivery) & $\begin{array}{l}\text { High (viral delivery)/low (nonviral } \\
\text { delivery) }\end{array}$ & Low (nonviral delivery) \\
\hline
\end{tabular}




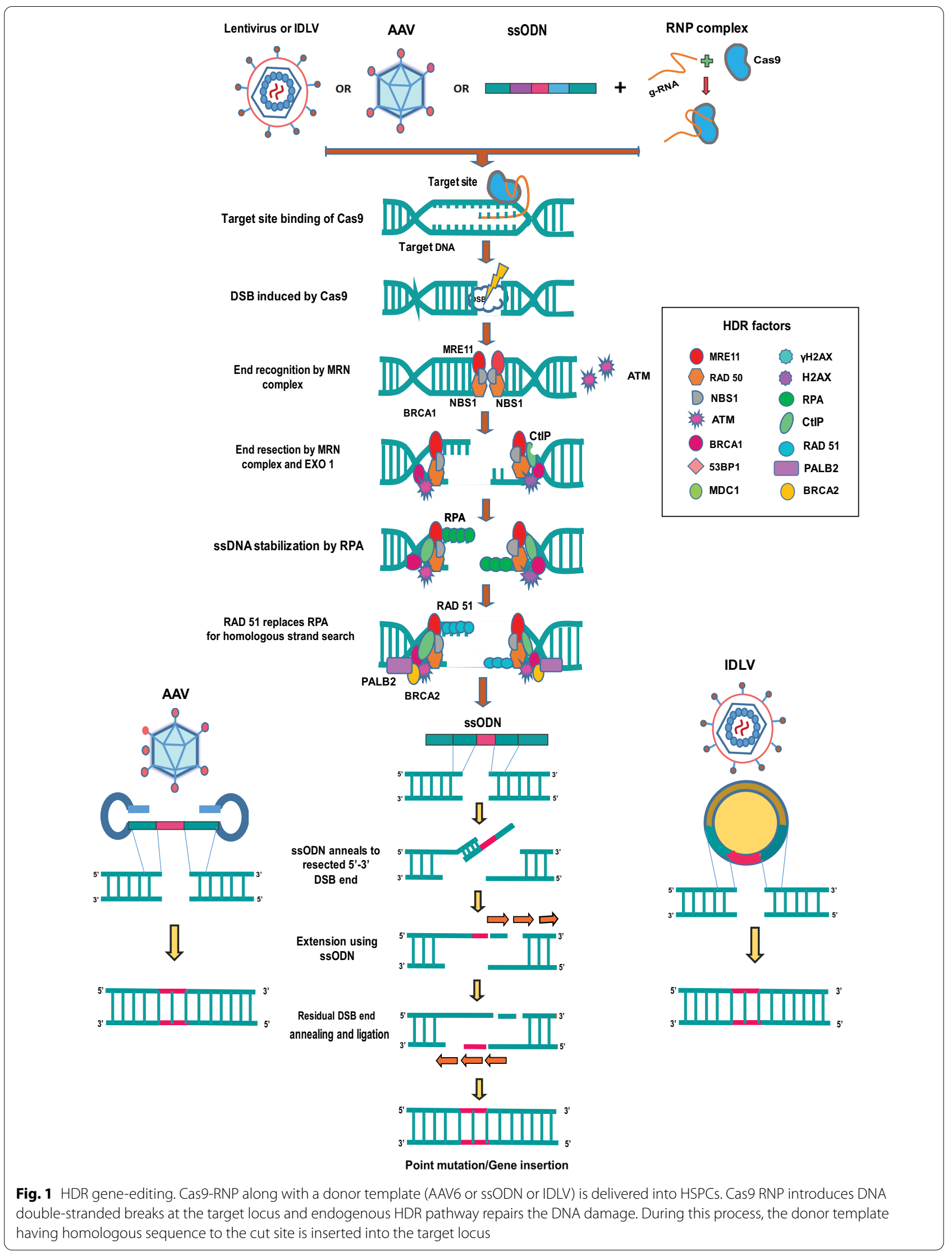


the D loop to cross-shaped structure known as holliday junction. The resolution of double holliday junction results in recombination such as either crossover or non-crossover with the help of nicking endonucleases. In SDSA pathway, the resected ssDNA undergoes strand invasion into a homologous duplex strand forming a D-loop and primes the synthesis of nascent DNA. The nascent DNA is then dissociated to anneal with the other resected strand. In BIR, one end of the DSB end is available for strand invasion and other end does not engage with the homologous sequence. This results in asynchronous leading and lagging strand synthesismediated new DNA. SSA is initiated when DSB happens between two repeated sequences that are oriented in same direction. SSA requires long end resection of $200 \mathrm{bp}$ and is independent of RAD51. RAD52 binds to the RPA coated ssDNA ends and anneals the resected single-stranded homologies to the DNA template. During the repair, single-stranded regions are made adjacent to the break which extends to the repeated sequences; thereby, complementary strands can anneal to each other. Finally, heterogenous flaps are removed by ERCC1-XPF nuclease and DNA gaps are ligated together. In SSTR pathway, the single-stranded DNA template gets annealed to the resected DNA strand and DNA extension is carried out with the help of DNA polymerase delta. The extended DNA product is now dissociated from the template, and the DNA ends are ligated using Ligase 1 or its analogues $[7,8]$. The resultant new strand of DNA is perfectly repaired using sequences from the template (Fig. 1).

\section{HDR gene editing}

Gene editing relies on engineered nucleases to recognize and cut specific DNA sequences and subsequently exploits the innate DNA machinery of the cell to repair the nuclease-induced DSB. Supplementation of homologous DNA template with site specific nucleases shifts the innate DNA repair pathway to HDR attaining targeted nucleotide changes. However, only cells in the S/G2-phases have phosphorylated CtIP to negate the antagonizing effect of 53BP1 on BRCA1 and thus respond to HDR donor supplementation. All HDR donor templates contain left (5') and right (3') homology sequences flanking the cut site, to exploit the HDR pathway. A wide range of viral derived or synthetically generated double-stranded and single-stranded HDR donor templates are currently tested for single nucleotide changes to large transgene insertion. Viral-derived HDR donors include integrase defective lentiviral vectors (IDLVs), adenovirus 5/35 serotype (AdVs), and adeno-associated vector (AAV) and single-stranded oligo deoxynucleotides (ssODNs) is a non-viral option.

\section{IDLV-HDR donor}

IDLVs were initially developed as alternatives to integrating viral vectors to avoid the risk associated with insertional mutagenesis. The D116 mutation within the catalytic domain of the integrase inactivates the integrase-mediated viral DNA integration and the resultant IDLV remains episomal which dissipates during cell divisions. This transient property makes IDLV a suitable HDR donor. IDLVs are free ended double-stranded DNA vectors, with the potential cargo delivery up to $10 \mathrm{~kb}$ [9]. IDLV templates have been tested in HSPCs for correcting SCID-X1 and SCD mutations. The results showed marginal levels of HDR $(2-18 \%)$ with post-transplant reduction in HDR frequency $(0.27-2 \%)$ [10-12]. The low frequency of target modification is due to the direct recombination of IDLVs with the target site before the initiation of HDR resulting in concatemer formation. In comparison with other HDR donors, IDLV donors exhibit reduced cytotoxic properties and it is presumed that the advancements in lentiviral transduction protocols such as cyclosporin $\mathrm{H}$ pre-treatment of HSPCs can be extended to IDLV donor templates for improving their efficiency [13].

\section{AdV HDR donor}

AdVs are dsDNA vectors with protein-capped ends. Commonly used AdV vectors are derived from the serotype 5 modified to allow insertion of larger transgene $(\sim 35 \mathrm{~kb})$. AdV exhibits broad range of tropism and high transduction efficiency [14]. Due to their large gene carrying capacity, they are used for delivery of the genes encoding the DNA cleaving endonucleases and the DNA templates for targeted insertion. The chimeric vector hybrid of serotypes 5 and 35 termed Ad5/35 has been used as HDR template, and it generates low efficiency HDR conversions $(\sim 2 \%)$ in cord blood (CB) HSPCs at HBB gene locus [12].

\section{AAV6 HDR donor}

Ever since the discovery, AAV vector has been an important component for gene therapy. However, the existence of antibodies against AAV limits the application in in vivo gene therapy. AAV vectors are designed for the incorporation of moderately large DNA transgene ( $5 \mathrm{~kb})$. AAV6 serotype, which exhibits tropism toward HSPCs, is extensively used as donor delivery vector. AAV6 donor templates are designed with long homology arms of $\sim 300-800 \mathrm{bp}$ and result in high frequency of HDR in HSPCs and several other primary cell types [15]. AAV6 is reported to show successful target insertion of 
reporter constructs up to 91\%, and AAV6 donors exhibit efficient HDR-mediated correction of sickle mutation than ssODN (50-60\% vs $29.6 \%$ ) in vitro, but the efficiency drops significantly in the long-term engrafted cells than the ssODN (32\% vs $17.5 \% \%$ ) [12, 16-18].

\section{sSODN-HDR donor}

ssODNs are short oligos often extending not more than 200 bp with homology arms in the range of 30-60 nucleotides flanking the desired nucleotide change. Compared to viral vectors, ssODNs possess several advantages such as simple design, reproducibility, short production time and relatively low cost. This makes them amenable to high-throughput applications. Asymmetric ssODNs (respective to the cut site) are efficient HDR templates than symmetric, and oligos complementary to non-target strand are highly effective as the Cas9 cleavage releases the 3' end of non-target strand making it amenable to ssODN. Asymmetric ssODN (PAM proximal nucleotide 91 and PAM distal nucleotides 36 flanking genomic breakpoint) facilitates HDR rates up to $60 \%$ in 293 -T cells $[12,15]$. However, such inferences on the ssODN design determining the HDR efficiency are not uniform across different cell types, loci and donors. Targeted corrections were obtained in HSPCs, in a wide range of loci like HBB and IL2RG [19]. HBB gene editing with ZFN and ssODN co-delivery results in HDR frequency of 5-15\%, and the efficiency decreased to $9 \%$ in the transplanted animals [12]. This indicates that optimized strategies must be developed for enhancing HDR rate by ssODNbased correction. ssODNs are the preferred substrates for SSTR, and the mechanism involved in ssODN-mediated repair is by direct annealing to the target DNA. ssODN approaches are beneficial for the correction of few nucleotides [15]. The major drawback of ssODN approach is that it cannot be applied for inserting a large transgene. Recently, Marson's group overcame this limitation by using a long ssODN and achieved the targeted integration of $>1 \mathrm{~kb}$ CAR construct with an efficiency up to $12.3 \%$ in the TRAC locus of the T-cells [20]. Such approach is yet to be tested in HSPCs.

\section{HDR in HSPC gene therapy}

Correction of defective gene by targeted gene manipulation is an attractive strategy in HSPC gene therapy. Editing of HSPCs provides long-term cure since these cells lie on the top of the hematopoietic hierarchy and effects can be retained in all the lineages, offering therapeutic benefits for both the hematological and immunological disorders. There are many studies showing correction of mutation using HDR-based gene editing in HSPCs for gene therapy application (Table 2).

\section{Blood disorders}

$\beta$-Hemoglobinopathies: SCD and $\beta$-thalassemia are the defects associated with the production of $\beta$-globin chains qualitatively (SCD) and quantitatively ( $\beta$-thalassemia) [21]. SCD arises due to a point mutation in the HBB exon (A $>\mathrm{T}$ or E6V), and mutation correction has been attempted with different types of HDR donor constructs. Nearly $50 \%$ of the E6V (HbS) alleles were reverted to wild type alleles by using RNP, AAV6 donor and selection of HDR cells. Similarly, 33\% correction was achieved in a RNP and ssODN-mediated selection/enrichment free strategy and up to $23.4 \%$ of corrected alleles were retained for a long term in engrafted hematopoietic stem cells [22]. Another study demonstrated the correction efficiency of $24.5 \pm 7.6 \%$ in vitro and $10 \%$ of corrected cells possessed long-term repopulation potential [23]. In line with this, targeted correction of $\beta$-thalassemia splicing variant IVS1-110 was achieved with Cas9 mRNA and ssODN with an efficiency of $8 \%$ [24]. Correction of wide range of $\beta$-thalassemia mutations requires personalized HDR-mediated gene correction approaches. As an alternative to mutation specific approach, a universal approach is developed which involves the reactivation of developmentally silenced $\gamma$-globin to compensate the decreased/defective $\beta$-globin chains. Several targets, such as $-175 \mathrm{~T}>\mathrm{C},-195 \mathrm{~T}>\mathrm{C}$ and $-113 \mathrm{~A}>\mathrm{G} \mathrm{HPFH}$ mutations, are known to induce $\gamma$-globin re-activation and are demonstrated successfully in erythroid cell lines K562 and HUDEP-2. These targets are yet to be tested in HSPCs [25].

Hemophilia: Liver-targeted gene therapy clinical trials with AAV vectors are showing promising outcomes for hemophilia, but the existence of antibodies against AAV limits the in vivo gene therapy application. As an alternate, ex vivo HSPC gene-editing approach, where $\alpha$-globin targeted integration of FIX-R338L transgene, was developed to express FIX in erythroid cells [26]. This indicates that HDR gene editing of HSPCs could also be used as a delivery system for therapeutic proteins.

Pyruvate kinase deficiency (PKD) disease: Mutations in the pyruvate kinase Isozymes R/L (PKLR) gene cause premature destruction of red blood cells resulting in PKD disease. Insertion of codon-optimized pyruvate kinase cDNA at its endogenous locus and puromycin selection of targeted cells to achieve $70 \%$ of gene-corrected cells is an exciting approach. However, poor in vivo engraftment of in vitro selected cells $(<1 \%$ HDR cells) remains a big obstacle to this approach [27].

\section{Primary immunodeficiency disorders}

$\mathrm{X}$-Linked chronic granulomatous disease (X-CGD): Mutations in the Cytochrome B-245 Beta Chain (CYBB) 
Table 2 Therapeutic HDR gene-editing strategy for genetic disorders using ZFNs, TALENs and Cas9

\begin{tabular}{|c|c|c|c|c|c|c|}
\hline Disease & Nuclease & Target locus & $\begin{array}{l}\text { HDR-gene-editing } \\
\text { strategy }\end{array}$ & Delivery route & Experimental model & References \\
\hline \multirow[t]{7}{*}{$\begin{array}{l}\text { Sickle cell disease \& } \\
\beta \text {-thalassemia }\end{array}$} & ZFNs & $\mathrm{HBB}$ & $\begin{array}{l}\text { ZFN mRNA and IDLV/ } \\
\text { ssODN }\end{array}$ & $\begin{array}{l}\text { Electroporation (Harvard } \\
\text { apparatus) }\end{array}$ & $\begin{array}{l}\text { HSPCs from healthy } \\
\text { donor and SCD patient }\end{array}$ & [10] \\
\hline & Cas9 & $\mathrm{HBB}$ & $\begin{array}{l}\text { Cas9 RNP and plasmid } \\
\text { donor }\end{array}$ & $\begin{array}{l}\text { Electroporation (Lonza } \\
\text { Nucleofector 4-D) }\end{array}$ & $\begin{array}{l}\text { HSPCs from healthy } \\
\text { donor and SCD patient }\end{array}$ & [23] \\
\hline & & & Cas9 mRNA and ssODN & $\begin{array}{l}\text { Electroporation (Neon } \\
\text { transfection system and } \\
\text { Lonza 4D Nucleofector) }\end{array}$ & $\begin{array}{l}\text { HSPCs from healthy } \\
\text { donor }\end{array}$ & [24] \\
\hline & & & Cas9 mRNA and IDLV & $\begin{array}{l}\text { Electroporation (ECM } \\
830 \text { Square wave elec- } \\
\text { troporator) }\end{array}$ & HSPCs from SCD patient & [53] \\
\hline & & & Cas9 RNP and ssODN & $\begin{array}{l}\text { Electroporation (Lonza } \\
\text { 4D Nucleofector) }\end{array}$ & $\begin{array}{l}\text { HSPCs from healthy } \\
\text { donor }\end{array}$ & [22] \\
\hline & & & Cas9 RNP and ssODN & $\begin{array}{l}\text { Electroporation (Lonza } \\
\text { 4D Nucleofector) }\end{array}$ & $\begin{array}{l}\text { HSPCs from healthy } \\
\text { donor }\end{array}$ & [54] \\
\hline & & & $\begin{array}{l}\text { Cas9 RNP and plasmid } \\
\text { donor }\end{array}$ & $\begin{array}{l}\text { Electroporation (Lonza } \\
\text { Nucleofector 2b) }\end{array}$ & HSPCs from SCD patient & [55] \\
\hline \multirow[t]{3}{*}{$X-S C I D$} & ZFNs & IL2RG & ZFN mRNA and IDLV & Electroporation (Lonza) & $\begin{array}{l}\text { HSPCs from healthy } \\
\text { donor and SCID-X1 } \\
\text { patient }\end{array}$ & [57] \\
\hline & & & ZFN and IDLV & Transduction & $\begin{array}{l}\text { HSPCs from healthy } \\
\text { donor }\end{array}$ & {$[58]$} \\
\hline & Cas9 & & Cas9 RNP and AAV6 & $\begin{array}{l}\text { Electroporation (Lonza } \\
\text { nucleofector 4D) }\end{array}$ & $\begin{array}{l}\text { HSPCs from SCID-X1 } \\
\text { patient }\end{array}$ & {$[30]$} \\
\hline $\begin{array}{l}\text { X-linked chronic granu- } \\
\text { lomatous disease }\end{array}$ & Cas9 & CYBB & Cas9 RNP and ssODN & $\begin{array}{l}\text { Electroporation (MaxCyte } \\
\text { systems) }\end{array}$ & $\begin{array}{l}\text { HSPCs from X-CGD } \\
\text { patient }\end{array}$ & [29] \\
\hline $\begin{array}{l}\text { Wiskott-Aldrich syn- } \\
\text { drome }\end{array}$ & Cas9 & WAS & Cas9 RNP and AAV6 & $\begin{array}{l}\text { Electroporation/trans- } \\
\text { duction (MaxCyte CTX } \\
\text { Flow electroporator) }\end{array}$ & $\begin{array}{l}\text { HSPCS from healthy } \\
\text { donor and WAS patient }\end{array}$ & {$[17]$} \\
\hline Mucopolysaccharidosis & Cas9 & CCR5 & Cas9 RNP and AAV6 & $\begin{array}{l}\text { Electroporation/trans- } \\
\text { duction (Lonza nucleo- } \\
\text { fector 4D) }\end{array}$ & $\begin{array}{l}\text { HSPCs from healthy } \\
\text { donor }\end{array}$ & [34] \\
\hline Gaucher disease & Cas9 & CCR5 & Cas9 RNP and AAV6 & $\begin{array}{l}\text { Electroporation/trans- } \\
\text { duction (Lonza 4D } \\
\text { nucleofector) }\end{array}$ & $\begin{array}{l}\text { HSPCs from healthy } \\
\text { donor }\end{array}$ & [59] \\
\hline $\begin{array}{l}\text { Pyruvate Kinase Defi- } \\
\text { ciency }\end{array}$ & TALEN\& Cas 9 & PKLR & $\begin{array}{l}\text { TALEN plasmid/Cas9 RNP } \\
\text { and plasmid donor }\end{array}$ & $\begin{array}{l}\text { Electroporation (Lonza } \\
\text { AMXA II nucleofection } \\
\text { system) }\end{array}$ & $\begin{array}{l}\text { HSPCs from healthy } \\
\text { donor }\end{array}$ & {$[27]$} \\
\hline IPEX syndrome & Cas9 & FOXP3 & Cas9 RNP and AAV6 & $\begin{array}{l}\text { Nucleofection/trans- } \\
\text { duction (Lonza 4D } \\
\text { nucleofector) }\end{array}$ & $\begin{array}{l}\text { HSPCs from healthy } \\
\text { donor }\end{array}$ & {$[32]$} \\
\hline
\end{tabular}

gene results in defective production of antimicrobial reactive oxygen species. CYBB gene mutation has been corrected with ZFNs coupled with AAV6 donors in CGD patient-derived HSPCs achieving 58\% HDR in vitro and $6-16 \%$ in vivo [28]. CRISPR/Cas9 RNP and ssODNmediated correction of $\mathrm{C} 676 \mathrm{~T}$ point mutation with an in vitro efficiency of $20 \%$ and in vivo efficiency of $13 \%$ was achieved [29].

$\mathrm{X}$-linked severe combined immunodeficiency (SCID$\mathrm{X} 1)$ : Mutations in $\gamma$ chain $(\gamma \mathrm{c})$ encoding interleukin 2 receptor subunit gamma (IL2RG) gene, a major subunit of encoding for common $\gamma$ chain $(\gamma \mathrm{c})$, leads to SCID-X1 [30]. Endogenous insertion of IL2RG cDNA using ZFN with IDLV or AAV6 was achieved with an efficiency of $10 \%$ \& $25 \%$ in wild-type and patient HSPCs, respectively [31]. Similarly, with CRISPR-Cas9 and AAV6 donors, the insertion frequency was achieved up to $45 \%$ in patient HSPCs in vitro and functional correction was achieved in $10-20 \%$ of LT-HSCs in vivo [30].

Wiskott-Aldrich syndrome (WAS): Mutations in WAS gene result in reduced levels of WAS protein. CRISPR/Cas9-mediated insertion of WAS cDNA into the endogenous site was achieved up to $47.9 \%$ in patient HSPCs resulting in restoration of WASp expression in $49 \%$ of the cells. In vivo studies showed the targeted 
insertion frequency of $40.7 \%$ and $37 \%$ in hCD $45+$ cells \& hCD19+B-cells, respectively [17].

Immune dysregulation, polyendocrinopathy, enteropathy, X-linked (IPEX) syndrome: IPEX syndrome occurs due to the mutations in the fox head box protein 3 (FOXP3) gene, a critical transcription factor necessary for regulatory T-cells (Tregs). Using the CRISPR-Cas9/ AAV6-mediated gene editing, FOXP3 cDNA was inserted into its endogenous locus and FOXP3 expression was restored in $29 \pm 8 \%$ of edited HSPCs that are capable of partially reconstituting Tregs $\left(\mathrm{CD} 4^{+} \mathrm{CD} 25^{+}\right.$ $\mathrm{FOXP}^{+}$) with retention of $60 \%$ edited cells in vivo [32].

\section{Metabolic disorders}

Mucopolysaccharidosis type I: Mutations in alphaL-iduronidase (IDUA) gene reduce the expression of IDUA causing glycosaminoglycan accumulation in lysosomes resulting the diseases [33, 34]. Targeted insertion of IDUA gene to the CCR5 safe harbor locus using AAV6 donors resulted in higher HDR frequencies up to $54 \% \pm 10$ and $44 \% \pm 7$ in CB and PB HSPCs, respectively, and a fivefold decrease in long-term engraftment of genemanipulated cells [34].

Gaucher disease: Mutations in glucocerebrosidase gene results in insufficient levels of glucocerebrosidase (GCase). In HSPCs, targeted insertion of glucocerebrosidase cassettes to the human CCR5 safe harbor locus using AAV6 donor templates resulted in HDR frequencies up to $51.5 \pm 9.1 \%$ [33]. This monocyte-/macrophagespecific expression strategy generated supraphysiologic GCase in vivo.

\section{Challenges with HDR gene editing in HSPCs}

Homology-directed repair holds a great potential in gene therapy as it allows precise customization of the genome, but this technique is not exempt from limitations. Although the gene-editing techniques have become well established through the years, HDR-based manipulation strategies, especially with HSCs, need rigorous improvization for successful clinical translation.

\section{Design and delivery of donor DNA template}

Unlike the naturally occurring homologous recombination where sister chromatids act as the correction template, HDR-mediated gene-editing experiments require exogenous donor DNA template. The donor template can be customized in a versatile manner for the incorporation of DNA modifications of single nucleotide to several kilobases. However, a strong synergy of the RNPs and ssODNs is required for efficient editing and the addition of donor template can possibly hinder the delivery potential. Importantly, the donor template requires extensive characterization of the length of the homologous arms, polarity, PAM shield and the backbone modifications for an efficient HDR [15].

\section{Toxicity of exogenous oligonucleotide templates}

A major challenge while using oligo donor is elicitation of immune response in the target cells. Oligo donors could mimic pathogen entry and the host cell, incapable of distinguishing both, activates Interferon gamma (IFNY) mediated inflammatory immune response leading to apoptosis. In addition, free/exposed DNA ends in ssODNs are prone to exonuclease attack, thereby reducing the availability of donor templates for HDR. While the capped DNA ends in AAV plasmids confer better stability, their larger size increases the nucleotide burden $[35,36]$ Gene-editing-mediated cellular toxicity is also triggered by p53 pathway, as they are the immediate responders to the DNA damage, leading to apoptosis and gradual diminishment of cells. The p53 activity is increased by the donor on the introduction of DNA templates generating more cytotoxicity than editing with RNP [37].

\section{Cell cycle restrictions}

HDR editing in HSPCs is challenging as the templated repair is permissive only during the S/G2 phases of the cell cycle. Hence, HSPCs and in particularly primitive HSCs, exhibit low susceptibility to HDR editing. While cytokine stimulation and prolonged culture are used to push HSPCs into cycling, these conditions are also associated with impairment in the engraftment of the HSPCs. The conventional cell cycle synchronization strategies are also observed to be challenging in HSPCs as there are no reports on the engraftment potential of synchronized HSPCs [38].

\section{Poor HDR editing efficiency in vitro}

The overall efficiency of HDR gene editing remains low than the non-template correction mechanisms-NHEJ \& MMEJ. While greater than 90\% NHEJ gene-editing efficiency can be achieved, there are no reports on HDR gene-editing efficiency of $>50 \%$. While selection of HDR positive cells can increase the frequency of HDR-edited cells, such a selection approach may not be possible with ssODN-based gene editing. Additionally, HDR-mediated large insertions occur at much lower rates than single nucleotide changes and small insertions. Also, different loci exhibit variabilities in the frequency of editing events due chromosome status and inherent bias toward NHEJ/ MMEJ $[39,40]$. 


\section{Reduced engraftment and long-term repopulating capacity in vivo}

While $<50 \%$ of HSPCs are undergoing HDR gene editing in vitro, the xeno-transplantation experiments have indicated an additional problem of reduction in the frequency of HDR edited cells in vivo. HDR editing showed disappointing results in transplantation assays, with dramatic reduction in the engraftment of HDR cells. This may be due to one of the following factors-HDR levels are higher in the progenitor population than in primitive HSCs/long-term repopulating cells resistant to the templated DNA repair/the cells that have undergone HDR lose stemness and are not retained in vivo. Thus, there is always a fundamental imbalance between HDR editing and the stemness of the HSPCs [30, 40-42].

\section{Scar in gene locus}

The HSPCs that were edited for HDR will contain a heterogenous pool of edited cells either homozygous, heterozygous or InDel only. In Some scenarios, the cells with InDels may be of concern. ssODN-mediated correction of SCD mutation in HBB locus results in correction of disease-causing mutation, but a fraction of cells with InDels can disrupt $\beta$-globin expression resulting in $\beta$-thalassemia like phenotype. Such unintended editing may not be a concern in many diseases. However, a precise edit is desirable [43].

\section{Strategies to overcome the limitations in HDR-based gene manipulation}

Despite the limitations and difficulties in HDR-mediated gene editing, their potency in targeted editing is promising. So, over the years, several strategies are explored for improving HDR editing.

\section{Inhibition of competitive NHEJ pathway}

Inhibition of proteins involved in different steps of the NHEJ repair pathway is a major approach to increase HDR efficiency. While stable knock-down of key repair proteins produce detrimental effects, usage of small molecules provides better solution of transient inhibition. SCR-7, NU7441, NU7026, STL127685, IC86621, M3814 and KU-0060648 are some of the small molecules tested to inhibit NHEJ and enhance the HDR. SCR-7 binds to the DNA-binding domain of ligase IV to inhibit NHEJ and improves HDR frequency in mammalian cell lines. Treatment with SCR7 resulted in an additive effect when combined with Adenovirus 4 (Ad4) E1B55K and E4orf6 proteins and improved the insertions of large transgene into Kell locus. NU7026, a DNA-dependent protein kinase (DNA-PK) inhibitor, improves HDR in iPSCs. Several other DNA-PK inhibitors like NU-7441 and
KU-0060648 increased plasmid and ssODN-mediated HDR in HEK 293 T cells. NU-7441 increased the AAV6 donor-mediated HDR efficiency in iPSCs. IC86621 and M3814 enhance the HDR in V3 cells and k562 cells, respectively. CRISPY Mix (a mixture of small molecules: trichostatin A, MLN4924, NU7026 and NSC 15520) is shown to increase the HDR in iPSCs $[5-7,19]$. Since most of the molecules for NHEJ inhibition have been tested in different human cell types but not in HSPCs, the above-mentioned molecules can be tested in HSPCs to enhance the HDR.

\section{Activation of HDR promoting factors}

L755507, resveratrol, brefeldin A, RS-1, MLN4924 and NSC 15520 are the few molecules tested for enhancing HDR. Both L755507 and resveratrol increased HDR efficiency in porcine fetal fibroblast by increasing the expression of the key HDR factors. In addition, L755507 and brefeldin promote insertion of large fragment into ACTA2 locus in mouse embryonic stem cells (mESCs). Farrerol, a herbal compound, is found to enhance the HDR rates in HEK293FT cells and mESCs [44]. RS-1 enhances HDR in HEK-293A and U2OS osteosarcoma cell lines by recruiting RAD51. MLN4924 increases the extent of DNA end resection at double-stranded break site and promotes HDR in iPSCs. NSC 15520 which blocks the interaction of RPA to RAD9 and to p53 increases the HDR in iPSCs $[5,6,19]$. Though several NHEJ-independent factors are employed to increase HDR, efficiency of the small molecules varies based on the cell types and gene locus. The functional repercussions of small molecule-treated gene-edited HSPCs need careful validation since it is highly unexplored. Concentration of small molecules and experimental conditions should be further optimized in order to achieve high HDR efficiency in HSPCs.

\section{Altering the chromatin state}

Chromatin state is shown to influence HDR gene editing and altering the chromatin state through modulation of HDAC activity, and histone octamer complex around the DNA affects the donor DNA integration. Entinostat (HDAC1/2/3 inhibitor) and panobinostat (Pan HDAC inhibitor) increase the HDR rate in HEK cell lines [45]. TSA, a HDAC $1 / 2$ inhibitor, increases the frequency of HDR by $40 \%$ when used with RNP and AAV6 donor [46]. Valproic acid (VPA), a Class I (HDAC1,2,3,8) and IIa (HDAC4,5,7,9) HDAC inhibitor, enhances the HDR in human embryonic stem cells [47]. Similarly, romidepsin (Class I, IIa, IIb (HDAC6,10) \& IV (HDAC11) and VPA increase the gene targeting efficiency in mESCs [48]. All these suggest that HDAC inhibition facilitates the Cas9 
access to target DNA and increases the gene-editing frequencies, thereby enhancing the HDR efficiency.

\section{Altering the HSPC cell cycle}

Cell cycle status influences the HDR outcomes, and one potential option is to increase the proportion of cells in $\mathrm{S}$ and $\mathrm{G} 2$ phases of the cell cycle. Restricting gene editing to S/G2 phase by Cas9-geminin fusion allowed transient Cas9 activity in S/G2 phase, thereby increasing the HDR/ NHEJ ratio. Simultaneous treatment of hGemCas9 and a Cyclin-dependent kinase 1 inhibitor RO-3306 showed an increase in HDR levels in HSPCs. XL-413, an inhibitor of Cycle 7-related protein kinase (CDC7), delayed the cells in S phase and increases the HDR. HSPCs were also allowed for a controlled cycling to increase the HDR by 5 - to 6 -fold, and then, quiescence was introduced with Rapamycin and CHIR99021. Nocodazole, a microtubule polymerization inhibitor, and aphidicolin, a G1/S phase inhibitor, increased the HDR rates in HEK 293 cells. Microtubule polymerization inhibitor ABT-751 increased the gene integration in HSPCs [5, 19]. Although different cell synchronizing strategies have been used in HSPCs, the effect of synchronization on the stemness, genetic stability and differentiation potential of stem cells needs further investigation.

\section{Lowering donor template toxicity}

Cell toxicity varies with the types of donor template used with the dsDNA, exhibiting higher toxicity than ssDNA oligos. Chemical modifications such as phosphorothiate (PS) modifications at the $3^{\prime}$ and $5^{\prime}$ end of the ssODN lower exonuclease attack, thus reducing cellular toxicity [35]. Since the naked DNA template is potentially toxic to the cells, use of chromatin DNA template which appears to be natural form of DNA lowers the template-associated toxicity [49]. Nuclease-induced DSB leads to the activation of p53 pathway thereby influences the survival of cells. Ameliorating the p53 mediated effects with the help of a dominant negative p53 protein helps to lower the donor template toxicity and improve the viability of edited HSPCs [37].

\section{Increasing donor availability at the target site}

Increase in global donor concentration is shown to have a direct impact on the HDR events but affects the viability of HSPCs. As an alternative, the donor DNA can be concentrated at the Cas 9 cleavage sites by physical linking of the ssODN to the Cas9 using various linkage chemistry. Cas9-ssODN conjugation by thiol-maleimide chemistry increased the HDR in INS-1E cells (Fig. 2a). Cas9 fusion proteins linked to the $\mathrm{O}^{6}$-benylguanine coupled ssODN
(Fig. 2b) increased the precise correction rates in HEK $293 \mathrm{~T}$ cells. ssODN covalently tethered to the Cas9-porcine circovirus 2 rep protein via fused $\mathrm{HUH}$ endonuclease increased the HDR in HEK293T (Fig. 2c). In S1mplex system, biotinylated ssODN donor linked to the gRNA, modified with streptavidin-aptamer complex, increased the HDR/indel ratio in HSPCs (Fig. 2d). Similarly, biotin modified ssODN is linked to the avidin fused Cas9 via flexible linker (Fig. 2e) achieving higher HDR frequency in mouse embryos (Fig. 2e). Testing these approaches in HSPCs could provide interesting results $[5,50]$.

\section{Global expression of HDR promoting factors}

HDR-enhancing factors have been extensively tested for application in gene editing, and different factors such as RAD51, RAD52, RAD54 and BRCA1 were overexpressed globally for HDR elevation. Coexpression of RAD51/RAD54 is shown to increase the HDR efficiency in HT-1080 cell lines. Similarly, the expression of either wild type or hyper-recombination variants of BRCA1 increased the HDR efficiency in HEK-293A cell lines. RAD52 motif protein 1 (RDM1) prevents the G2/M cell cycle arrest and thereby enhances the HDR frequency, and Cas9-RDM1 fusion variant is yet to be tested in human cells including HSPCs. Inhibition of 53BP1 by an ubiquitin variant (i53) enhanced the HDR efficiency with both AAV6 and ssODN donor. Co-expression of both factors, dn53BP1 and RAD 52, increased the frequency of HDR at multiple loci in HEK 293 cells and iPSCs. Overall, this technology can be applied in HSPCs to improve HDR and achieve higher rates of gene correction. Though several strategies are employed to achieve global expression of HDR promoting factors, further improvements must be necessary to achieve successful HDR-based gene therapy in HSPCs $[4,51,52]$.

\section{Conclusion}

The availability of innovative tools presents the scope for next-generation therapeutics for the treatment of genetic disorders. The rapid evolution of gene-editing tools and cutting-edge CRISPR/Cas9 technology has allowed development of more efficient and feasible gene therapy approaches than viral vectors. Reports from NHEJ mediated gene-editing approach showed promising results in the on-going clinical trials. HDR gene editing has not yet reached the clinical studies owing to the low efficiency and the complexities involved. The recent technologies such as base editing and prime editing provide a strong challenge to HDR editing as their efficiency is observed to be superior to HDR gene editing. However, the IND approvals for 
A
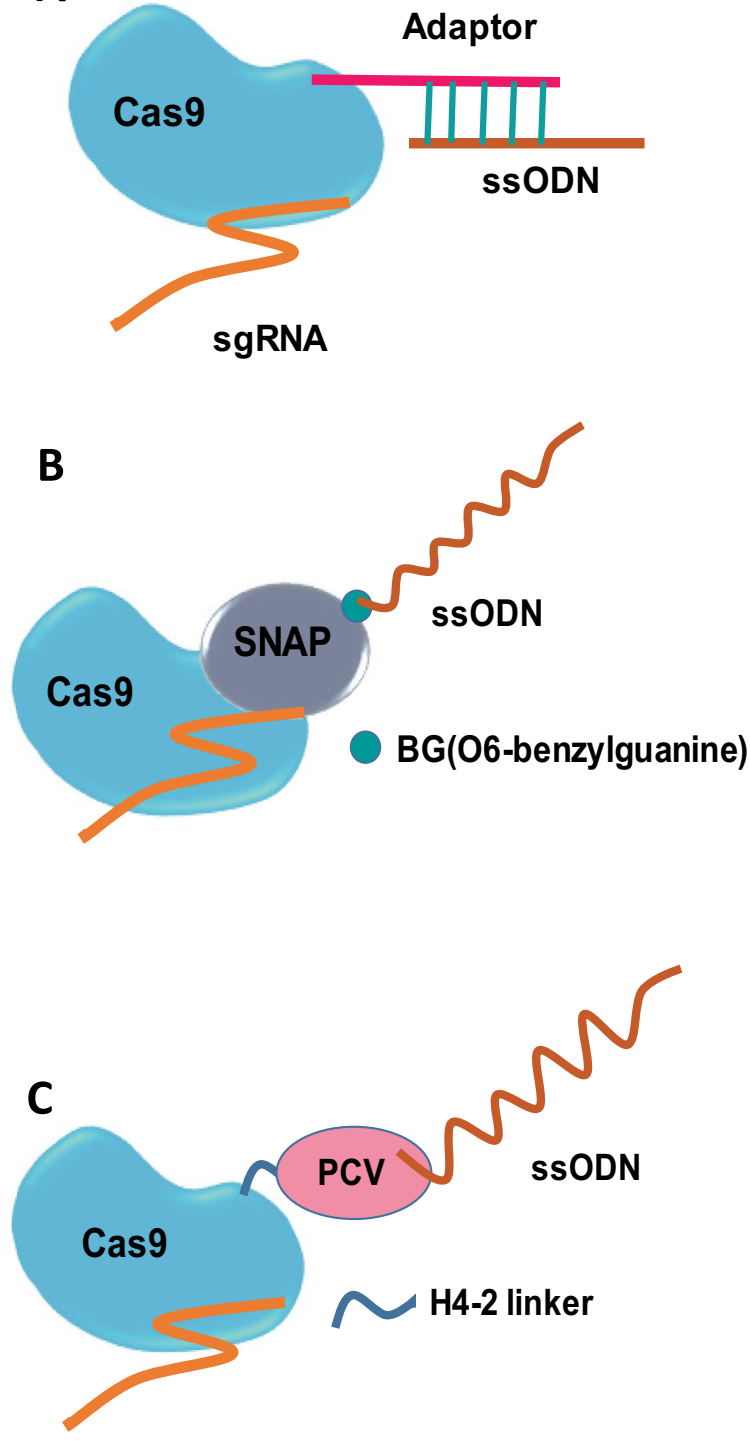
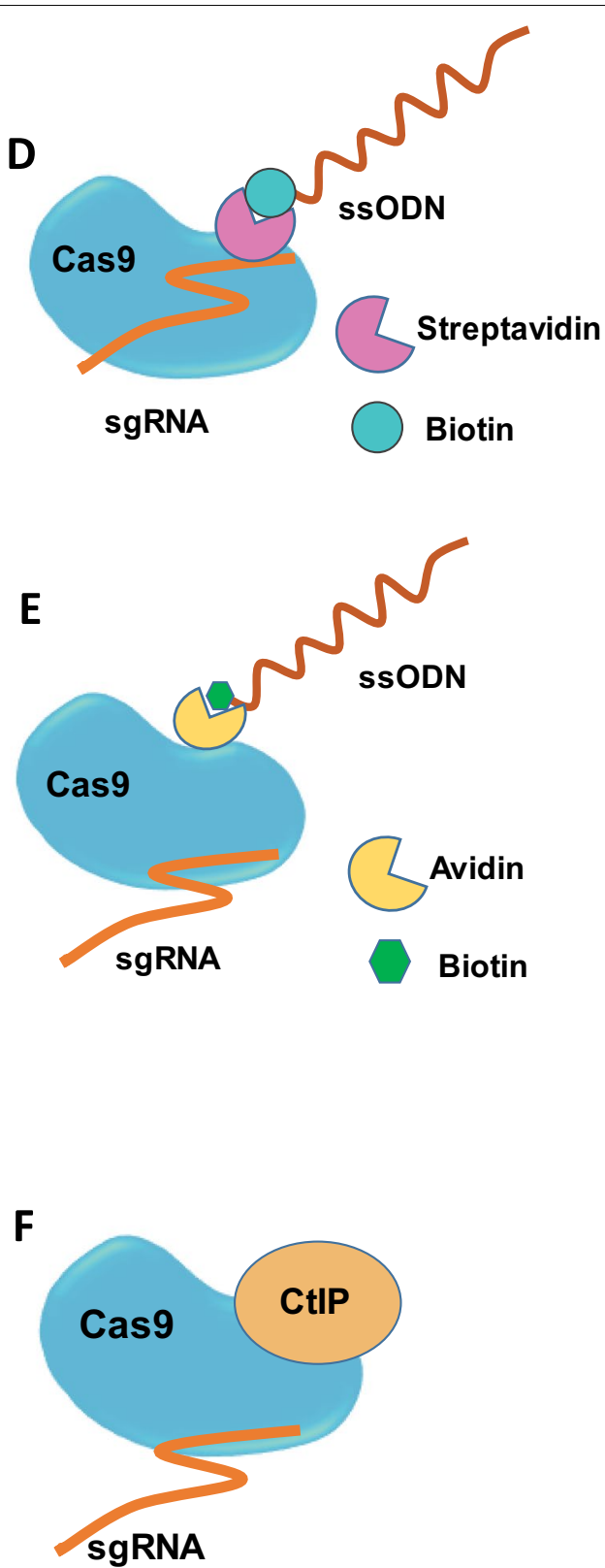

Fig. 2 Strategies for increasing the donor availability at Cas9 cut site. a Thiol-maleimide chemistry-based Cas9 and ssODN conjugation. A short oligonucleotide adaptor is attached to the Cas9 via thiol-maleimide and appended with ssODN by base pairing. b Cas9-ssODN linking using snap-tag technology. Snap-tag fused Cas9 is covalently linked to the BG coupled ssODN. c RNP-ssODN tethering using HUH endonuclease. Cas9 is fused to the HUH endonuclease PCV Rep protein via H4-2 linker. Then, ssODN is covalently attached to the HUH endonuclease PCV. $\mathbf{d}$ In S1 mplex system, sgRNA modified using streptavidin-binding aptamer is linked to the biotinylated ssODN, forming RNP-ssODN complex. e Cas9 is fused to the avidin via flexible linker and Cas9 is linked to biotin modified ssODN. f Direct fusion of CtIP and Cas9 nuclease

UCSF/UCLA approach of Cas9-ssODN-mediated SCD correction and Standford university approach of Cas9AAV6-mediated SCD correction provide lots of hope for HDR gene editing. With the success of HDR-based gene editing, we are expected to witness the success of targeted gene therapy for several genetic disorders in future. Alongside the technical research and advancement for gene therapy, it is also important to design a cost-effective treatment module so that the therapy is accessible to most patients, especially in developing countries. 


\begin{abstract}
Abbreviations
AAVs: Adeno-associated vectors; AdVs: Adenovirus vectors; BIR: Break-induced replication; BRCA1: Breast cancer type 1; CDNA: Complementary DNA; CRISPRCas9: Clustered regularlyy interspaced short palindromic repeats-associated RNA-guided Cas9; DNA-PKcs: DNA-dependent protein kinase catalytic subunit; DSB: Double-stranded break; FOXP3: Fox head box protein 3; HAT: Histone acetyltransferase; HBB: Hemoglobin subunit beta; HbS: Hemoglobin S; HDAC Histone deacetylase; HDR: Homology-directed repair; HSPCs: Hematopoietic stem and progenitor cells; IDLVs: Integrase defective lentiviral vectors; IPEX: Immune dysregulation, polyendocrinopathy, enteropathy, X-linked; iPSCs: Induced pluripotent stem cells; MMEJ: Microhomology-mediated end joining; NADPH: Nicotinamide adenine dinucleotide phosphate oxidase 2 (NOX2); NHEJ: Non-homologous end joining; SDSA: Synthesis-dependent stranded annealing; SSA: Single-stranded annealing; SsODNs: Single-stranded deoxynucleotides; SSTR: Synthesis-dependent stranded annealing; TALENs: Transcription activator-like nucleases; ZFNs: Zinc finger nucleases.
\end{abstract}

\section{Acknowledgements}

We thank Abisha Crystal Christopher, Karthik V Karuppusamy and Annlin George for critical reading of this manuscript.

\section{Authors' contributions}

MK \& ST designed the review outline. MK, PB \& ST performed the literature search and data analysis. MK, PB, VV and ST wrote the manuscript. All authors read and approved the final manuscript.

\section{Funding}

This work is funded by Department of Biotechnology, Government of India (BT/PR31616/MED/31/408/2019).

\section{Availability of data and materials \\ Not applicable.}

\section{Declarations}

Ethics approval and consent to participate

Not applicable.

\section{Consent for publication}

Not applicable.

\section{Competing interest}

The authors declare that they have no conflict of interest.

\section{Author details}

${ }^{1}$ Centre for Stem Cell Research (CSCR), a Unit of InStem Bengaluru, Christian Medical College Campus, Vellore, Tamil Nadu, India. ${ }^{2}$ Manipal Academy of Higher Education, Manipal, Karnataka, India.

\section{Received: 11 May 2021 Accepted: 19 August 2021}

Published online: 09 September 2021

\section{References}

1. Prakash V, Moore M, Yáñez-Muñoz RJ. Current progress in therapeutic gene editing for monogenic diseases. Mol Ther. 2016;24:465-74.

2. Li H, Yang Y, Hong W, et al. Applications of genome editing technology in the targeted therapy of human diseases: mechanisms, advances and prospects. Signal Transduct Target Ther. 2019. https://doi.org/10.1038/ s41392-019-0089-y.

3. Van Tendeloo VFI, Van Broeckhoven C, Berneman ZN. Gene therapy: Principles and applications to hematopoietic cells. Leukemia. 2001;15:523-44.

4. Liu M, Rehman S, Tang X, et al. Methodologies for improving HDR efficiency. Front Genet. 2019;10:1-9.

5. Yang H, Ren S, Yu S, et al. Methods favoring homology-directed repair choice in response to crispr/cas9 induced-double strand breaks. Int J Mol Sci. 2020;21:1-20.
6. Bischoff N, Wimberger S, Maresca M, et al. Improving precise CRISPR genome editing by small molecules: is there a magic potion? Cells. 2020:9:1-16.

7. Yeh CD, Richardson CD, Corn JE. Advances in genome editing through control of DNA repair pathways. Nat Cell Biol. 2019;21:1468-78.

8. Devkota S. The road less traveled: Strategies to enhance the frequency of homology-directed repair (HDR) for increased efficiency of CRISPR/Casmediated transgenesis. BMB Rep. 2018;51:437-43.

9. Banasik MB, McCray PB. Integrase-defective lentiviral vectors: Progress and applications. Gene Ther. 2010;17:150-7.

10. Hoban MD, Cost GJ, Mendel MC, et al. Correction of the sickle cell disease mutation in human hematopoietic stem/progenitor cells. Blood. 2015:125:2597-604.

11. Genovese P, Schiroli G, Escobar G, et al. Targeted genome editing in human repopulating haematopoietic stem cells. Nature. 2014:510:235-40

12. Romero Z, Lomova A, Said S, et al. Editing the sickle cell disease mutation in human hematopoietic stem cells: comparison of endonucleases and homologous donor templates. Mol Ther. 2019;27:1389-406.

13. Bauer DE, Pai SY. Getting past HSC security: cyclosporine $\mathrm{H}$ gives lentiviruses an entry pass. Cell Stem Cell. 2018;23:775-6.

14. Zhang $\mathrm{W}$, Chen $\mathrm{H}$, Zheng $\mathrm{X}$, et al. Targeted genome correction by a single adenoviral vector simultaneously carrying an inducible zinc finger nuclease and a donor template. J Biotechnol. 2014;188:1-6.

15. Salsman J, Dellaire G. Precision genome editing in the CRISPR era. Biochem Cell Biol. 2017;95:187-201.

16. Pattabhi S, Lotti SN, Berger MP, et al. In Vivo outcome of homologydirected repair at the HBB gene in HSC using alternative donor template delivery methods. Mol Ther Nucleic Acids. 2019;17:277-88.

17. Rai R, Romito M, Rivers $E$, et al. Targeted gene correction of human hematopoietic stem cells for the treatment of Wiskott-Aldrich Syndrome. Nat Commun. 2020. https://doi.org/10.1038/s41467-020-17626-2.

18. Wang J, Exline CM, Declercq JJ, et al. Homology-driven genome editing in hematopoietic stem and progenitor cells using ZFN mRNA and AAV6 donors. Nat Biotechnol. 2015;33:1256-63.

19. Salisbury-Ruf CT, Larochelle A. Advances and obstacles in homologymediated gene editing of hematopoietic stem cells. J Clin Med. 2021;10:513

20. Roth TL, Puig-saus $C$, Yu R, et al. Viral genome targeting. Nature. 2019;559:405-9.

21. Cavazzana M, Antoniani C, Miccio A. Gene therapy for B-hemoglobinopathies. Mol Ther. 2017;25:1142-54.

22. Magis W, DeWitt MA, Wyman SK, et al. In vivo selection for corrected B-globin alleles after CRISPR/Cas9 editing in human sickle hematopoietic stem cells enhances therapeutic potential. bioRxiv.

23. Park SH, Lee CM, Dever DP, et al. Highly efficient editing of the $\beta$-globin gene in patient-derived hematopoietic stem and progenitor cells to treat sickle cell disease. Nucleic Acids Res. 2019;47:7955-72.

24. Antony JS, Latifi N, Haque AKMA, et al. Gene correction of HBB mutations in CD34+ hematopoietic stem cells using Cas9 mRNA and ssODN donors. Mol Cell Pediatr. 2018:5:1-7.

25. Wienert B, Funnell APW, Norton $L$, et al. Editing the genome to introduce a beneficial naturally occurring mutation associated with increased fetal globin. Nat Commun. 2015;6:1-8.

26. Pavani $G$, Laurent $M$, Fabiano A, et al. Ex vivo editing of human hematopoietic stem cells for erythroid expression of therapeutic proteins. Nat Commun. 2020. https://doi.org/10.1038/s41467-020-17552-3.

27. Quintana-Bustamante O, Fañanas-Baquero S, Orman I, et al. Gene editing of PKLR gene in human hematopoietic progenitors through $5^{\prime}$ and $3^{\prime}$ UTR modified TALEN mRNA. PLOS ONE. 2019:14:1-20.

28. De Ravin SS, Reik A, Liu PQ, et al. Targeted gene addition in human CD34 + hematopoietic cells for correction of X-linked chronic granulomatous disease. Nat Biotechnol. 2016;34:424-9.

29. De Ravin SS, Li L, Wu X, et al. CRISPR-Cas9 gene repair of hematopoietic stem cells from patients with $X$-linked chronic granulomatous disease. Sci Transl Med. 2017. https://doi.org/10.1126/scitransImed.aah3480.

30. Pavel-Dinu M, Wiebking V, Dejene BT, et al. Gene correction for SCID-X1 in long-term hematopoietic stem cells. Nat Commun. 2019. https://doi.org/ 10.1038/s41467-019-09614-y.

31. Schiroli G, Ferrari S, Conway A, et al. Preclinical modeling highlights the therapeutic potential of hematopoietic stem cell gene editing for 
correction of SCID-X1. Sci Transl Med. 2017. https://doi.org/10.1126/scit anslmed.aan0820.

32. Lee E, Goodwin M, Lakshmanan U, et al. Gene editing using CRISPR enables FOXP3 gene repair in HSPCs and IPEX patient T cells. Cytotherapy. 2020;22:S20.

33. Scharenberg SG, Poletto E, Lucot KL, et al. Engineering monocyte/macrophage-specific glucocerebrosidase expression in human hematopoietic stem cells using genome editing. Nat Commun. 2020. https://doi. org/10.1038/s41467-020-17148-x.

34. Gomez-Ospina N, Scharenberg SG, Mostrel N, et al. Human genomeedited hematopoietic stem cells phenotypically correct Mucopolysaccharidosis type I. Nat Commun. 2019;10:1-14.

35. Renaud JB, Boix C, Charpentier M, et al. Improved genome editing efficiency and flexibility using modified oligonucleotides with TALEN and CRISPR-Cas9 nucleases. Cell Rep. 2016;14:2263-72.

36. Hanlon KS, Kleinstiver BP, Garcia SP, et al. High levels of AAV vector integration into CRISPR-induced DNA breaks. Nat Commun. 2019;10:1-11.

37. Schiroli G, Conti A, Ferrari S, et al. Precise gene editing preserves hematopoietic stem cell function following transient p53-mediated DNA damage response. Cell Stem Cell. 2019;24:551-565.e8.

38. Gerlach M, Kraft T, Brenner B, et al. Efficient knock-in of a point mutation in porcine fibroblasts using the CRISPR/Cas9-GMNN fusion gene. Genes Basel. 2018. https://doi.org/10.3390/genes9060296.

39. Zhu Z, González F, Huangfu D. The iCRISPR platform for rapid genome editing in human pluripotent stem cells. Methods Enzymol. 2014. https:// doi.org/10.1016/B978-0-12-801185-0.00011-8.

40. Zhang JP, Li XL, Li GH, et al. Efficient precise knockin with a double cut HDR donor after CRISPR/Cas9-mediated double-stranded DNA cleavage. Genome Biol. 2017;18:1-18.

41. Shin JJ, Schröder MS, Caiado F, et al. Controlled cycling and quiescence enables efficient HDR in engraftment-enriched adult hematopoietic stem and progenitor cells. Cell Rep. 2020. https://doi.org/10.1016/j.celrep.2020. 108093.

42. Mohrin M, Bourke E, Alexander D, et al. Hematopoietic stem cell quiescence promotes error-prone DNA repair and mutagenesis. Cell Stem Cell. 2010;7:174-85.

43. Métais JY, Doerfler PA, Mayuranathan T, et al. Genome editing of HBG1 and HBG2 to induce fetal hemoglobin. Blood Adv. 2019;3:3379-92.

44. Zhang W, Chen Y, Yang J, et al. A high-throughput small molecule screen identifies farrerol as a potentiator of CRISPR/Cas9-mediated genome editing. Elife. 2020;9:1-25.

45. Liu B, Chen S, La RA, et al. Inhibition of histone deacetylase 1 (HDAC1) and HDAC2 enhances CRISPR/Cas9 genome editing. Nucleic Acids Res. 2020;48:517-32.

46. Fu Y-W, Dai X-Y, Wang W-T, et al. Dynamics and competition of CRISPRCas9 ribonucleoproteins and AAV donor-mediated NHEJ, MMEJ and HDR editing. Nucleic Acids Res. 2021;49:1-17.
47. Takayama K, Igai K, Hagihara Y, et al. Highly efficient biallelic genome editing of human ES/PPS cells using a CRISPR/Cas9 or TALEN system. Nucleic Acids Res. 2017:45:5198-207.

48. Park H, Shin J, Choi H, et al. Valproic acid significantly improves CRISPR/ Cas9-mediated gene editing. Cells. 2020. https://doi.org/10.3390/cells 9061447

49. Cruz-Becerra G, Kadonaga JT. Enhancement of homology-directed repair with chromatin donor templates in cells. Elife. 2020;9:1-12.

50. Aird EJ, Lovendahl KN, St Martin A, et al. Increasing Cas9-mediated homology-directed repair efficiency through covalent tethering of DNA repair template. Commun Biol. 2018. https://doi.org/10.1038/ s42003-018-0054-2.

51. Tang XD, Gao F, Liu MJ, et al. Methods for enhancing clustered regularly interspaced short palindromic repeats/Cas9-mediated homologydirected repair efficiency. Front Genet. 2019. https://doi.org/10.3389/ fgene.2019.00551.

52. Benitez EK, Lomova Kaufman A, Cervantes L, et al. Global and local manipulation of DNA repair mechanisms to alter site-specific gene editing outcomes in hematopoietic stem cells. Front Genome Ed. 2020:2:601541

53. Hoban MD, Lumaquin D, Kuo CY, et al. CRISPR/Cas9-mediated correction of the sickle mutation in human CD34+ cells. Mol Ther. 2016;24:1561-9.

54. DeWitt MA, Magis W, Bray NL, et al. Selection-free genome editing of the sickle mutation in human adult hematopoietic stem/progenitor cells. Sci Transl Med. 2016. https://doi.org/10.1126/scitranslmed.aaf9336.

55. Dever DP, Bak RO, Reinisch A, et al. CRISPR/Cas9 $\beta$-globin gene targeting in human haematopoietic stem cells. Nature. 2016:539:384-9.

56. Martin RM, lkeda K, Cromer MK, et al. Highly efficient and marker-free genome editing of human pluripotent stem cells by CRISPR-Cas9 RNP and AAV6 donor-mediated homologous recombination. Cell Stem Cell. 2019:24:821-828.e5.

57. Genovese P, Schiroli G, Escobar G, et al. Targeted genome editing in human repopulating haematopoietic stem cells. Nature. 2014. https:// doi.org/10.1038/nature13420

58. Lombardo A, Genovese P, Beausejour CM, et al. Gene editing in human stem cells using zinc finger nucleases and integrase-defective lentiviral vector delivery. Nat Biotechnol. 2007;25:1298-306.

59. Gomez-Ospina N, Scharenberg SG, Lucot KL, et al. Monocyte lineagespecific glucocerebrosidase expression in human hematopoietic stem cells: a universal genome editing strategy for Gaucher disease. Mol Genet Metab. 2020;129:S64-5.

\section{Publisher's Note}

Springer Nature remains neutral with regard to jurisdictional claims in published maps and institutional affiliations.
Ready to submit your research? Choose BMC and benefit from:

- fast, convenient online submission

- thorough peer review by experienced researchers in your field

- rapid publication on acceptance

- support for research data, including large and complex data types

- gold Open Access which fosters wider collaboration and increased citations

- maximum visibility for your research: over $100 \mathrm{M}$ website views per year

At BMC, research is always in progress.

Learn more biomedcentral.com/submissions 